[12] W. Dorner, P. Demont et D. Chavannes. Microbiologie laitière, $1945 ; 193$.

[13] I. Saenz Lascano Ruiz. Le dosage du lactose dans les laits altérés par fermentation lactique. Les eonditions d'hydrolyse quantitative du lactose dans un lactoserum. Ann. Fals. Fraudes, 1954, 167 et 285.

[14] S. J. RowLAND. The determination of the nitrogen distribution in milk. J. Dairy Res., 9, 1938, 42.

\title{
DES DANGERS QUE SEMBLE COMPORTER L'UTILISATION DE L'ALCOOL ISOAMYLIQUE DANS LES LABORATOIRES D'ANALYSES DE LAITERIES
}

\author{
par \\ RAPHAEL MOREAU
}

L'alcool isoamylique $\mathrm{CH}_{\mathrm{CH}^{3}}^{\mathrm{CH}^{3}}>\mathrm{CH}-\mathrm{CH}^{2}-\mathrm{CH}^{2} \mathrm{OH}$ est couramment employé dans les laboratoires de laiterie qui, pour la détermination de la teneur en matière grasse des laits, crèmes et fromages, utilisent la méthode butyrométrique "Gerber" ou des méthodes analogues.

Le rôle de cet alcool est de détruire l'émulsion de la matière grasse. Lors de chaque analyse un volume de $1 \mathrm{~cm}^{3}$ est introduit dans le butyromètre au moyen d'une ampoule, munie d'un robinet doseur.

La manipulation de cet alcool ne présentait jusqu'à ces derniers temps aucun danger en raison des faibles quantités mises en œuvre par suite du nombre relativement restreint des analyses effectuées journellement, en raison aussi de la nature de l'appareil mesureur employé.

La généralisation du paiement du lait suivant sa richesse en matière grasse et la disparition du beurre fermier ont augmenté, dans des proportions très importantes, le nombre quotidien des analyses effectuées dans les usines laitières (plusieurs centaines par jour et par usine au lieu de plusieurs dizaines). Cette situation nouvelle a incité certains constructeurs de matériel de laboratoire à concevoir des doseurs multiples permettant d'introduire l'automatisme dans l'application des méthodes butyrométriques et de réaliser ainsi des gains de temps importants.

Malheureusement, certains appareils doseurs à tubes plongeurs présentent, par suite de leur conception, le grave inconvénient de favoriser l'évaporation de l'alcool isoamylique dont les vapeurs se répandent dans l'atmosphère.

D'autre part, toutes les précautions ne sont pas toujours prises, lors des manipulations, pour éviter cette évaporation. Les personnes 
qui, occasionnellement, pénètrent dans un laboratoire peu spacieux, insuffisamment ventilé, où un appareil de ce genre se trouve en service, sont incommodées au bout de quelques minutes. Aux toussotements succède une gêne respiratoire et, souvent, le visiteur est victime d'un malaise ou d'un trouble qu'on ne peut définir et qui pourrait être dû au pouvoir narcotique de l'alcool isoamylique.

Le personnel des laboratoires de laiteries, vraisemblablement par suite de l'accoutumance de l'organisme, n'est pas incommodé immédiatement. Au bout d'un laps de temps qui dépend de l'individu, il arrive que le manipulateur se trouve dans un état nauséeux, et parfois les migraines apparaissent.

L'action toxique de l'alcool isoamylique n'est peut-être plus à démontrer, mais il semble qu'elle ne soit guère connue de ceux qui l'emploient. C'est pourquoi il ne nous paraît pas inutile de relater l'expérience sommaire que nous avons réalisée afin de nous rendre compte, par nous-mêmes, de l'influence que pouvait exercer l'alcool isoamylique sur l'animal.

Un cobaye pesant 330 grammes a été placé à différentes reprises pendant 8 heures sous une cloche d'un volume approximatif de 19 litres. Au sommet de cette cloche une ouverture de 35 millimètres de diamètre rendait impossible toute confination de l'atmosphère. Sous cette cloche ont été placés, en même temps, des tampons de coton imbibés d'alcool isoamylique. Les tampons ont été pesés au début et à la fin de l'expérience afin de déterminer la quantité d'alcool évaporé. Cette quantité s'est élevée à $0 \mathrm{~g}$. 250 , représentant un volume gazeux de 0 1. 063 pour la durée d'expérience de 8 heures indiquée ci-dessus.

Le cobaye présenta d'abord des signes d'excitation, puis, rapidement, une dyspnée s'établit. Le rythme respiratoire, d'abord rapide, se ralentit ensuite jusqu'à la fin de l'expérience. A ce moment, le cobaye fut sorti de la cloche; il semblait prostré, ses réflexes étaient amoindris. Il ne retrouva son aspect habituel que plusieurs heures après.

Par contre, un autre cobaye, placé pendant le même laps de temps sous une cloche identique, mais sans tampons imbibés d'alcool isoamylique, ne présenta aucun symptôme anormal.

Dans une autre expérience, le cobaye mourut et l'autopsie ne révéla aucune lésion viscérale nette. La mort n'a donc pas été provoquée par l'asphyxie, comme les signes de dyspnée auraient pu le faire croire, mais sans doute par paralysie du système nerveux.

D'après une expérience que nous avons effectuée sur nousmêmes, il semble que l'intoxication comporte deux phases : au 
cours de la première phase, l'action de l'alcool isoamylique provoque une accélération des battements du cœur, il s'agit de la phase d'excitation. Au cours de la seconde phase, un ralentissement du rythme respiratoire est observé.

L'alcool isoamylique détruit les émulsions de la matière grasse par abaissement de la tension superficielle, tel est son rôle dans les analyses de lait et de crème. N'est-il pas permis alors de se demander s'il n'aurait pas une action du même ordre sur le système nerveux ? Evidemment, seules des recherches plus approfondies pourraient permettre d'élucider l'action de cet alcool sur l'organisme.

Il serait très intéressant de connaître la concentration limite dans l'atmosphère pouvant être supportée sans inconvénient. Aussi il nous paraît indispensable que les conditions dans lesquelles l'alcool isoamylique peut être employé dans les laboratoires de laiteries, fassent l'objet d'une étude sérieuse et que cette étude soit suivie d'une règlementation si cela s'avère nécessaire, comme nos observations le laissent supposer.

Si nous avons cru utile de relater ces observations, malheureusement trop sommaires, c'est parce qu'il nous a semblé opportun de poser un problème dont la solution intéresse la santé de tout le personnel employé dans les laboratoires d'analyses des laiteries.

\title{
APPAREIL A PROJECTION POUR LA LECTURE PRÉCISE DES BUTYROMETRES (1)
}

\author{
par \\ JEAN PIEN
}

L'une des conditions primordiales de l'exactitude des résultats obtenus dans le dosage de la matière grasse du lait par la méthode Gerber est la précision de la lecture du nombre de divisions occupé par la colonne grasse.

Or de nombreuses causes d'erreur peuvent intervenir à ce stade :

1) Déplacement spontané de la colonne grasse: dès que le butyromètre est sorti du bain-marie à $65^{\circ}$, le liquide hydro-acide contenu dans la panse se refroidit, se contracte, et le niveau occupé par la colonne grasse tend à descendre. En se déplaçant, la colonne grasse tapisse l'intérieur du tube gradué d'un film gras qui ne se rassemble pas immédiatement dans la couche grasse principale et diminue d'autant la longueur apparente de cette colonne.

(1) Communication au XIVe Congrès International de Laiterie (Rome, septembre 1956). 